\title{
Parietal bone osteomyelitis in melioidosis
}

\author{
Hariprasad Sadanand Shetty, ${ }^{1}$ Ajay Raj Mallela, ${ }^{1}$ Barkur Ananthakrishna Shastry, ${ }^{2}$ \\ Vasudeva Acharya ${ }^{2}$
}

${ }^{1}$ Department of Internal Medicine, Kasturba Medical College, Manipal University, Manipal, Karnataka, India

${ }^{2}$ Department of Medicine, Kasturba Medical College, Manipal University, Manipal, Karnataka, India

Correspondence to Dr Ajay Raj Mallela, ajayprakashdr@gmail.com

Accepted 7 February 2015
To cite: Shetty HS, Mallela AR, Shastry BA, et al. BMJ Case Rep Published online: [please include Day Month Year] doi:10.1136/bcr-2014208612

\section{SUMMARY}

We report a case of a 55-year-old man with uncontrolled diabetes who presented with pneumonia. During his hospital stay his clinical status worsened and he had a focal seizure. MRI showed central nervous system involvement and parietal bone osteomyelitis. As the patient's blood culture and endotracheal aspirate grew Burkholderia pseudomallei, melioidosis was diagnosed. He was treated with meropenem after failure to respond to ceftazidime. He gradually improved over a period of 4 weeks and was discharged. Early diagnosis and therapy resulted in improved outcome.

\section{BACKGROUND}

Infection with Burkholderia pseudomallei causes melioidosis. Melioidosis is predominantly seen in northern Australia and southeast Asia, including India. ${ }^{1}$ Common modes of transmission are percutaneous inoculation, inhalation, aspiration and ingestion. Common clinical manifestations of the disease are pneumonia and skin infection. Central nervous system (CNS) involvement is, however, rare; its manifestations are severe and diverse ranging from cerebral abscess to meningoencephalitis with flaccid paraparesis. To the best of our knowledge, only one case of parietal bone osteomyelitis has been reported in the literature. ${ }^{2}$ Rarity of CNS involvement in melioidosis and raising awareness among treating physicians about the condition is the driving factor for reporting this case.

\section{CASE PRESENTATION}

A 55-year-old man with uncontrolled diabetes mellitus and hypertension presented with fever and cough with expectoration of 15 days' duration. This was associated with worsening dyspnoea for 5 days. On examination, the patient was febrile with bilateral basal crackles. He was suspected to have pneumonia and was empirically started on a third generation cephalosporin and macrolide (ceftriaxone with azithromycin). The patient's haemodynamic condition deteriorated (he developed hypotension with tachycardia) and he was shifted to the intensive care unit (ICU). He had severe respiratory distress with tachypnoea and was therefore intubated and put on ventilatory support. During his ICU stay he developed tonic clonic movements of the left upper limb, which was diagnosed as simple partial seizure, and he was started on antiepileptic medication. CT imaging of the brain was inconclusive. As the patient continued to have persistent fever and refractory seizures, MRI of the brain was carried out. The MRI showed subdural empyema with early parietal bone osteomyelitis. Blood and endotracheal (ET) aspirate showed growth of $B$. pseudomallei on culture, which confirmed the diagnosis of melioidosis.

\section{INVESTIGATIONS}

On blood investigation, the patient's blood sugars were found to be high (fasting $320 \mathrm{mg} / \mathrm{dL}$ and postprandial $460 \mathrm{mg} / \mathrm{dL}$ ) with glycosylated haemoglobin of $10.9 \%$ (normal 4-6.5\%), which was suggestive of uncontrolled diabetes mellitus. Complete blood picture and other biochemical investigations were within normal limits. Chest X-ray showed bilateral lower zone non-homogenous infiltrates with air bronchograms suggestive of pneumonia. CT of the brain showed no abnormality. Cerebrospinal fluid (CSF) analysis was uninformative. MRI of the brain showed a peripherally enhancing subdural collection in right parietal convexity (figure 1) and areas of restricted diffusion in right temporal and parietal bone showing heterogeneous marrow enhancement on contrast injection, which raised the possibility of early osteomyelitic

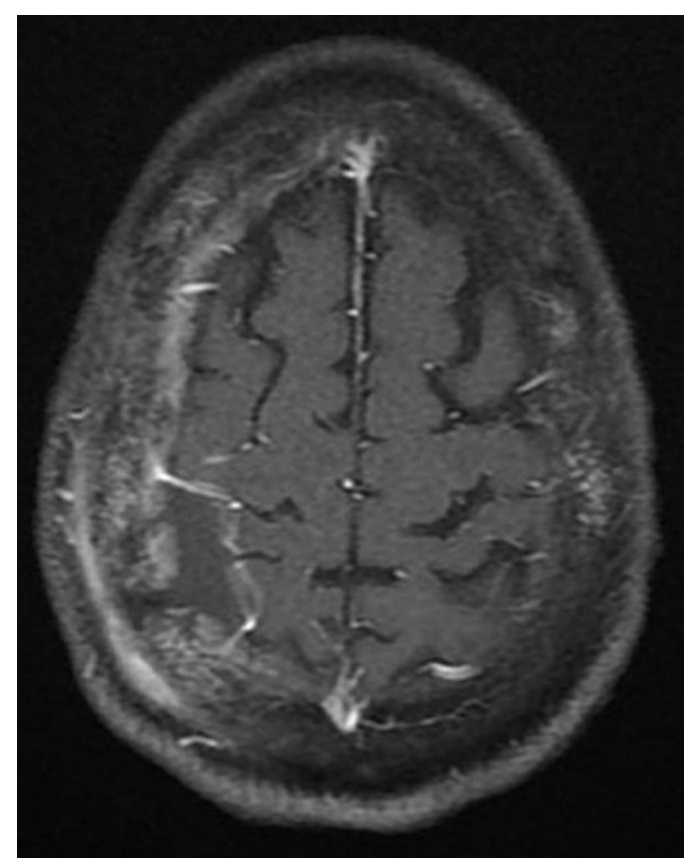

Figure 1 Axial T1 fatsat postcontrast image showing peripherally enhancing subdural collection (empyema) along the right parietal convexity with enhancement of right parietal bone. 
changes (figure 2). Right temporal lobe and insular cortex showed areas of subtle hyperintensities with contrast enhancement, which were suggestive of early cerebritis.

Blood culture and ET aspirate grew B. pseudomallei.

Three samples of sputum for acid-fast bacilli were negative.

\section{DIFFERENTIAL DIAGNOSIS}

Initially, the patient was suspected to have pneumonia. Disseminated tuberculosis was also thought as a possibility in light of the persistent fever, worsening of clinical status and focal seizures. CT imaging ruled out the possibility of tuberculoma and intracranial space occupying lesion. CSF analysis did not show any evidence of herpes simplex encephalitis. Based on MRI findings and culture sensitivity, the report of infection with B. pseudomallei was confirmed.

\section{TREATMENT}

The patient was started on intensive therapy with intravenous ceftazidime $2 \mathrm{~g}$ given every 6 hours. As the patient did not respond to the therapy, he was switched after 4 days to intravenous meropenem $2 \mathrm{~g}$ every 8 hours. Seizures were managed with multiple antiepileptic medication (levetiracetam, phenytoin, valproate sodium and oxcarbazepine). Trimethoprimsulfamethoxazole (TMP-SMX) was added to the intensive therapy at a dose $8 / 40 \mathrm{mg} / \mathrm{kg}$ orally twice daily after 2 weeks. A neurosurgical opinion was sought for drainage of subdural empyema but the patient was advised medical management. His blood glucose was managed with insulin. Eradication therapy following intensive therapy was planned for a duration of 6 months with continuation of TMP-SMX at a dose of $8 / 40 \mathrm{mg} /$ kg orally twice daily.

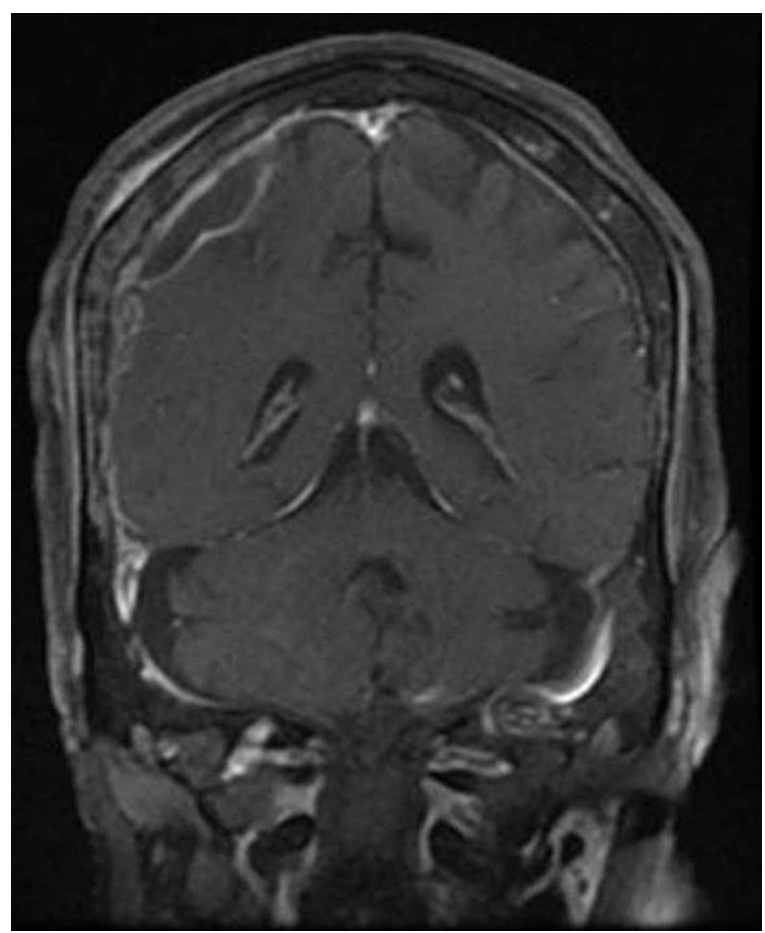

Figure 2 Coronal T1-weighted postcontrast image showing extra-axial collection along the right parietal convexity with marrow enhancement of right parietal bone suggestive of early osteomyelitic changes.

\section{OUTCOME AND FOLLOW-UP}

The patient gradually improved after 2 weeks of intensive therapy. Repeat chest X-ray showed clearing of pneumonic patch and repeat blood culture showed no growth. The patient was extubated and shifted to the ward once his clinical status improved. He had no further episode of seizure and antiepileptic medication was optimised. Eight weeks of intensive therapy were planned of which 4 weeks were completed during his hospital stay. MRI of the brain, which had been performed during follow-up after 2 months of intensive therapy, showed reduction in the size of subdural empyema (figure 3) and the patient was continued on eradication therapy (TMP-SMX). He was advised to follow-up after 2 months.

\section{DISCUSSION}

Melioidosis with neurological manifestations is associated with significant morbidity and mortality. Early literature mentions inhalation as the primary route but now percutaneous inoculation is considered the main mode of transmission of disease. ${ }^{3}$ In our case, inhalation could be the primary route with haematogenous spread. Most important risk factors are diabetes, alcohol abuse and chronic kidney disease. ${ }^{4} 5$ The incubation period of disease is about 9-21 days. ${ }^{6}$ Pneumonia was found to be the most common manifestation of disease in all studies. ${ }^{1} 478$ Osteomyelitis as presenting symptom was seen only in $3 \%$ of patients. ${ }^{4-11}$ Osteomyelitis is a very rare manifestation of the disease, its aetiology being considered to be a disseminated infection affecting metaphysis of long bones. ${ }^{9}$ This case report highlights the rare presentation of early parietal bone osteomyelitis with subdural empyema associated with melioidosis. A previous study by Chadwick et $a l^{12}$ showed cerebral abscess to be the source of infection for osteomyelitis of the parietal bone. Laboratory testing includes all available samples for culture as it is the mainstay for diagnosis. Patients presenting with neurological manifestations should be investigated with CT

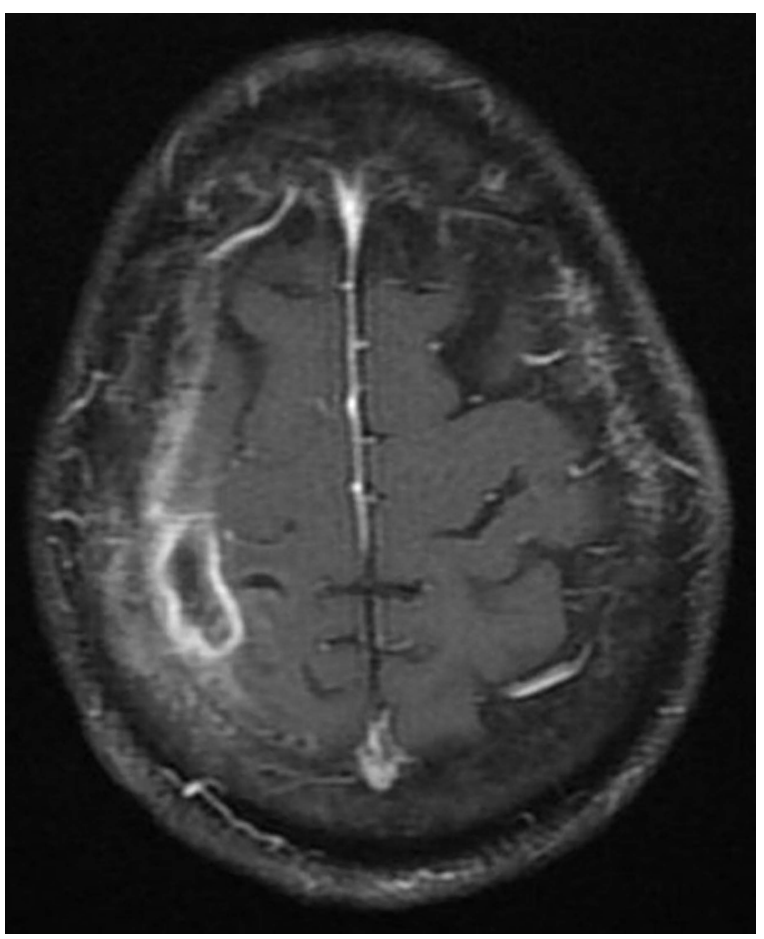

Figure 3 Follow-up MRI axial T1 fatsat postcontrast image showing reduction in size of the right parietal subdural empyema. 
of the brain to rule out intracranial lesions related to disease, however, CT of the brain may be normal in patients with encephalomyelitis, so MRI would be a better option. Patients diagnosed to have melioidosis should be managed with an initial intensive phase and eradication phase whether infection is mild or severe. Recommended drugs are parenteral ceftazidime $50 \mathrm{mg} / \mathrm{kg}$ (up to $2 \mathrm{~g}$ daily) intravenously every 6 hours or meropenem $25 \mathrm{mg} / \mathrm{kg}$ (up to $1 \mathrm{~g}$ daily) intravenously every 8 hours for intensive therapy followed by TMP-SMX $8 / 40 \mathrm{mg} / \mathrm{kg}$ (up to $320 / 1600$ ) orally every 12 hours either alone or in combination with doxycycline for eradication therapy. Ceftazidime has become the drug of choice for intensive therapy based on the results of a randomised trial carried out in Thailand. ${ }^{13}$ In our case, the patient improved with meropenem; previous comparative studies showed better efficacy of carbapenems over ceftazidime in severe melioidosis. ${ }^{14}{ }^{15}$ Drug penetration to the brain is considered a major concern in neurological melioidosis; ceftazidime and carbapenem both achieve appropriate concentrations in the brain during the acute phase of disease. Addition of cotrimoxazole with parenteral ceftazidime in the intensive phase has not shown additional benefit but is still considered, in view of its excellent tissue penetration in clinical conditions such as bone, joint and neurological melioidosis. ${ }^{16}{ }^{17}$ As per the literature, the duration of the intensive phase for disseminated melioidosis is $4-8$ weeks and for the eradication phase it is 3 or 6 months. ${ }^{18}$ A prospective study by Sarovich $e t$ al ${ }^{19}$ has demonstrated that recurrent melioidosis is no longer common and showed the shift of relapse to reinfection. This decreased relapse was attributed to better antibiotic cover and prolonged intensive phase therapy. In our case, in view of the peculiar site of the osteomyelitis (close to the leptomeninges), and minimal bone damage and good clinical response to antimicrobial therapy, the patient was managed conservatively.

\section{Learning points}

- Parietal bone osteomyelitis with subdural empyema is an unusual central nervous system (CNS) presentation in melioidosis.

- Aggressive management of CNS melioidosis requires intensive therapy of at least $4-8$ weeks followed by eradication therapy for 6 months.

- Burkholderia pseudomallei should be considered in the differential diagnosis in immunocompromised patients with febrile illness and pneumonia.

- Lack of awareness about melioidosis in non-endemic regions will misdiagnose the organism.
Contributors ARM and HSS contributed to the preparation of manuscript and management of the patient. BAS and VA were involved in the management of the patient.

\section{Competing interests None.}

Patient consent Obtained.

Provenance and peer review Not commissioned; externally peer reviewed.

\section{REFERENCES}

1 Currie BJ, Dance DA, Cheng AC. The global distribution of Burkholderia pseudomallei and melioidosis: an update. Trans R Soc Trop Med Hyg 2008;102 (Suppl 1):S1-4.

2 Miksić NG, Alikadić N, Lejko TZ, et al. Osteomyelitis of parietal bone in melioidosis. Emerg Infect Dis 2007;13:1257-9.

3 Currie BJ, Fisher DA, Howard DM, et al. The epidemiology of melioidosis in Australia and Papua New Guinea. Acta Trop 2000;74:121-7.

4 Currie BJ, Ward L, Cheng AC. The epidemiology and clinical spectrum of melioidosis: 540 cases from the 20 year Darwin prospective study. PLoS Negl Trop Dis 2010:4:e900.

5 Currie BJ, Jacups SP, Cheng AC, et al. Melioidosis epidemiology and risk factors from a prospective whole-population study in northern Australia. Trop Med Int Health 2004;9:1167-74.

6 Currie BJ, Fisher DA, Anstey NM, et al. Melioidosis: acute and chronic disease, relapse and re-activation. Trans R Soc Trop Med Hyg 2000;94:301-4.

7 Currie BJ. Melioidosis: an important cause of pneumonia in residents of and travellers returned from endemic regions. Eur Respir I 2003;22: $542-50$.

8 Simpson AJ, Suputtamongkol Y, Smith MD, et al. Comparison of imipenem and ceftazidime as therapy for severe melioidosis. Clin Infect Dis 1999;29:381-7.

9 Subhadrabandhu T, Prichasuk S, Sathapatayavongs B. Localised melioidotic osteomyelitis. J Bone Joint Surg Br 1995;77:445-9.

10 Popoff I, Nagamori J, Currie B. Melioidotic osteomyelitis in northern Australia. Aust N Z J Surg 1997;67:692-5.

11 Morse LP, Smith J, Mehta J, et al. Osteomyelitis and septic arthritis from infection with Burkholderia pseudomallei: a 20-year prospective melioidosis study from northern Australia. J Orthop 2013;10:86-91.

12 Chadwick DR, Ang B, Sitoh YY, et al. Cerebral melioidosis in Singapore: a review of five cases. Trans R Soc Trop Med Hyg 2002;96:72-6.

13 White NJ, Dance DA, Chaowagul W, et al. Halving of mortality of severe melioidosis by ceftazidime. Lancet 1989;2:697-701.

14 Smith MD, Wuthiekanun V, Walsh AL, et al. Susceptibility of Pseudomonas pseudomallei to some newer beta-lactam antibiotics and antibiotic combinations using time-kill studies. J Antimicrob Chemother 1994;33:145-9.

15 Cheng AC, Fisher DA, Anstey NM, et al. Outcomes of patients with melioidosis treated with meropenem. Antimicrob Agents Chemother 2004;48:1763-5.

16 Chierakul W, Anunnatsiri S, Short JM, et al. Two randomized controlled trials of ceftazidime alone versus ceftazidime in combination with trimethoprim-sulfamethoxazole for the treatment of severe melioidosis. Clin Infect Dis 2005;41:1105-13.

17 Chierakul W, Anunnatsiri S, Chaowagul W, et al. Addition of trimethoprim-sulfamethoxazole to ceftazidime during parenteral treatment of melioidosis is not associated with a long-term outcome benefit. Clin Infect Dis 2007:45:521-3.

18 Writing Group. Melioidosis. In: Therapeutic guidelines: antibiotic. 14th edn. North Melbourne: Therapeutic Guidelines Ltd, 2010.

19 Sarovich DS, Ward L, Price EP, et al. Recurrent melioidosis in the Darwin Prospective Melioidosis Study: improving therapies mean that relapse cases are now rare. J Clin Microbiol 2014;52:650-3.

Copyright 2015 BMJ Publishing Group. All rights reserved. For permission to reuse any of this content visit http://group.bmj.com/group/rights-licensing/permissions.

BMJ Case Report Fellows may re-use this article for personal use and teaching without any further permission.

Become a Fellow of BMJ Case Reports today and you can:

- Submit as many cases as you like

- Enjoy fast sympathetic peer review and rapid publication of accepted articles

- Access all the published articles

- Re-use any of the published material for personal use and teaching without further permission

For information on Institutional Fellowships contact consortiasales@bmjgroup.com

Visit casereports.bmj.com for more articles like this and to become a Fellow 